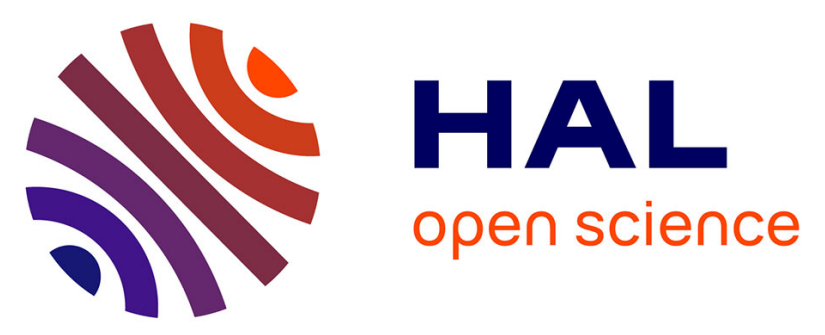

\title{
Contraction Control of a Fleet Circular Formation of AUVs under Finite Communication Range
}

\author{
Lara Briñon Arranz, Alexandre Seuret, Carlos Canudas de Wit
}

\section{To cite this version:}

Lara Briñon Arranz, Alexandre Seuret, Carlos Canudas de Wit. Contraction Control of a Fleet Circular Formation of AUVs under Finite Communication Range. ACC 2010 - American Control Conference, Jun 2010, Baltimore, Maryland, United States. pp.6. hal-00448036

\section{HAL Id: hal-00448036 https://hal.science/hal-00448036}

Submitted on 18 Jan 2010

HAL is a multi-disciplinary open access archive for the deposit and dissemination of scientific research documents, whether they are published or not. The documents may come from teaching and research institutions in France or abroad, or from public or private research centers.
L'archive ouverte pluridisciplinaire HAL, est destinée au dépôt et à la diffusion de documents scientifiques de niveau recherche, publiés ou non, émanant des établissements d'enseignement et de recherche français ou étrangers, des laboratoires publics ou privés. 


\section{Contraction Control of a Fleet Circular Formation of AUVs under Limited Communication Range}

\author{
Lara Briñón Arranz* \\ lara.brinon-arranz@inrialpes.fr \\ INRIA Rhône-Alpes* \\ NeCS Team, Grenoble, France
}

\author{
Alexandre Seuret** \\ alexandre.seuret@gipsa-lab.inpg.fr
}

\author{
Carlos Canudas de Wit** \\ carlos.canudas-de-wit@gipsa-lab.inpg.fr
}

\author{
CNRS, Department of Automatic Control, \\ GIPSA-Lab** \\ NeCS Team, Grenoble, France
}

\begin{abstract}
This work proposes a novel control algorithm dedicated to multi-agent systems with nonlinear dynamics. The aim of this paper is for the agents to form a circular formation whose center is fixed and whose radius is given by a time-varying reference. The problem of uniform distribution of all the agents along the circle is also addressed under the assumption of limited communication range. This communication constraint is tackled by using a cooperative control scheme which includes the Laplacian matrix of the communication graph (distancedependent). The multi-agent system is simulated with Matlab. Videos showing the simulations are accessible though Web ${ }^{1}$.
\end{abstract}

\section{INTRODUCTION}

Control of multi-agent systems has received a lot of attention in recent years [1], [2]. Particulary, the problem of collective or cooperative control of an autonomous systems group is an important developing field motivated by a number of application in many areas including cooperative control of underwater and unmanned air vehicles (AUVs and UAVs) [3], [4], consensus [1], [5], formation control [6], [7], flocking [8], distributed sensor networks [2], [9] and rendezvous [10]. Formation control and motion coordination have been extensively studied. The reader can refer to [2], [11], [12], [13], [7], [14] among many others. These studies concern circular and parallel formations [2], [7], [11], [14], but also motions of formation induced by flocking [8], [15].

This paper focusses on the problem of design control strategies for a class of non linear multi-agent systems to achieve circular formation. Interesting results have been already proposed in [2] and [11] to deal with a time-invariant problem, when the circular formation has a fixed center and a constant radius. A relevant problem is now to relax these constraints and to consider time-varying formations. This problem belongs to the fundamental transformations of circular formations [16]. These transformations are pertinents to some applications where the agents should perform collaborative tasks requiring the formation to displace towards an a priori unknown direction and adjust to some particular form. For instance, in source seeking applications, the formation should displace in the source gradient direction and contract its size to adapt to the level curves of the source plume.

\footnotetext{
${ }^{1}$ Simulations are accessibles in the CONNECT project web at
} http://www.lag.ensieg.inpg.fr/connect/
In the context of the source seeking for underwater vehicles, it is relevant to constrain the agents in an appropriate shape, for instance circular, to avoid unnecessary energy waist. Moreover, ensuring that the agents are uniformly distributed along the circle might be more adequate to produce efficient search motions.

Another difficulty in the underwater fleet formation problem is due to the information transmission in a marine media. Communication between agents is confronted with several difficulties such as signal distorsion and interference, doppler effect, etc. Communication in shallow water amplifies these limitations. In this work, we assume that the communication between each agent is "good enough" within a particular range specific to the application. Therefore, the previously described contraction formation control will be studied under such a limited-range communication assumption, where the communication graph depends on the agent's relative position [1], [5], [17].

Note that a first contribution concerning the translation of a circle is proposed in [18]. However the case of contraction (or, equivalently, expansion) of a circular formation is still an open problem. In the present work, a control law is developed so that the formation is able to track a time-varying radius reference. An additional component of the control law is also added to achieved the uniform distribution of the agents along the time-varying circle. Moreover, this collaborative control law stands for the case of range-dependent graph, and provides some simulations showing the asymptotic convergence.

The paper is organized as follows. The following section recalls an existing results of the field of circular formation control and shows their limitations. Section III exposes the main contribution of the article which deals with the contraction and the expansion of the formation. Section IV proposes an extension to achieve the uniform distribution for the communication constraint described above. Simulations of multi-agent systems tracking a circular formation with a time-varying radius are given in Section V.

Notation. A complex number $\mathbf{z}$ is written in boldface and is expressed as $\mathbf{z}=x+i y$ where $i^{2}=-1$ and where $x=\operatorname{Re}\{z\}$ and $y=\operatorname{Im}\{z\}$ correspond to the real and 


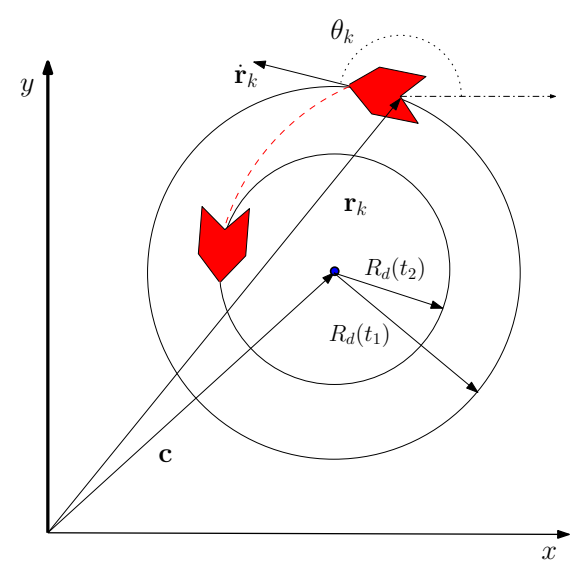

Fig. 1. Illustration of the problem formulation.

the imaginary part of $\mathbf{z}$. The notations $|\mathbf{z}|=\langle\mathbf{z}, \mathbf{z}\rangle^{1 / 2}$ and $\angle \mathbf{z}$ stand for the magnitude and the argument of the complex number $\mathbf{z}$. Denote $\left\langle\mathbf{z}_{1}, \mathbf{z}_{2}\right\rangle$ the product $\operatorname{Re}\left\{\overline{\mathbf{z}}_{1}^{T} \mathbf{z}_{2}\right\}$, where $\overline{\mathbf{z}}_{1}^{T}$ represents the conjugate transpose of $\mathbf{z}_{1}$. Note that the real part (respectively the imaginary part) of a complex number $\mathbf{z}$ can be written as $\langle\mathbf{z}, 1\rangle$ (and respectively $\langle\mathbf{z}, i\rangle$ ). Thus, for any complex functions $\mathbf{z}_{1}$ and $\mathbf{z}_{2}$, the equality $\left\langle\mathbf{z}_{1}, i\right\rangle\left\langle\mathbf{z}_{2}, 1\right\rangle-\left\langle\mathbf{z}_{1}, 1\right\rangle\left\langle\mathbf{z}_{2}, i\right\rangle=\left\langle\mathbf{z}_{1}, i \mathbf{z}_{2}\right\rangle$ holds.

This paragraph presents some basic tools of graph theory. When an agent $k$ communicates with an agent $j$ both agents are called neighbors. The set of neighbors of agent $k$ is denoted by $\mathcal{N}_{k}$. The communication topology for the groups of agents can be represented by means of a graph $G(V, E)$ where $V=\{1,2, \ldots, N\}$ is the set of vertices (agents) and $E=\left\{(k, j): j \in \mathcal{N}_{k}\right\}$ the set of edges (communication links) such that $(k, j) \in E$ if agent $k$ communicates with agent $j$.

\section{PROBLEM FORMULATION AND PRELIMINARIES}

\section{A. Model of agents}

Consider a set of $N$ agents (vehicles) whose dynamics are given by the standard agent model commonly used in the literature to model AUVs restricted kinematics [2], [6], [7], [11], [14], [19] and [20]:

$$
\begin{aligned}
& \dot{\mathbf{r}}_{k}=v_{k} e^{i \theta_{k}} \\
& \dot{\theta}_{k}=u_{k}
\end{aligned}
$$

where $k=1, \ldots, N, \mathbf{r}_{k}$ and $\theta_{k}$ represent the position vector and the heading angle, as illustrated in Figure 1. The variables $v_{k}, u_{k}$ are the control inputs. This model corresponds to a kinematic unicycle fitting with model properties subject to a simple nonholonomic constraint.

The problem considered here is to design a control law such that the group of AUVs forms a circle whose center is fixed and whose radius traks a time-varying reference $R_{d}(t)$ as described in Figure 1. $R_{d}(t)$ is considered here as an external reference.

\section{B. Preliminaries}

Some previous works on the field of coordinated control and specifically on planar collective motions, use the kinematic model in which each vehicle moves in the plane subject to planar steering control, which is our model (1) with constant velocity $v_{k}=v_{0}=1, \forall k$. In [2], the authors suggest a control law to converge to a circular formation center at a particular and constant $\mathbf{c}$. It corresponds to the center of mass and is obtained by solving a consensus algorithm. The control law uses the relative position vector from the center to vehicle $k$ defined as $\tilde{\mathbf{r}}_{k}=\mathbf{r}_{k}-\mathbf{c}=\frac{1}{N} \sum_{j=1}^{N}\left(\mathbf{r}_{k}-\mathbf{r}_{j}\right)$. For such a formation, the authors propose the following theorem:

Theorem 1 (Leonard et al. [2]) Consider the vehicle model (1) with $v_{k}=v_{0}=1, \forall k$. Then the control law:

$$
u_{k}=\omega_{0}\left(1+\kappa\left\langle\tilde{\boldsymbol{r}}_{k}, \dot{\boldsymbol{r}}_{k}\right\rangle\right)
$$

where $\kappa>0$ and $\left|\omega_{0}\right| \neq 0$, ensures that all the agents converge to a circular formation centered at $\boldsymbol{c}$ and of radius $R_{0}=v_{0} /\left|\omega_{0}\right|$.

\section{Fundamental limitations}

These previous results are only applicable to the case of a fixed formation center $\mathbf{c}$, and a constant radius $R_{0}$. In this situation, it is sufficient to design a control law such that the velocity of all the agents is constant (for instance $\left.v_{k}=1, \forall k\right)$. However, when it comes to the case of a time-varying radius $R_{d}(t)$, a limitation appears naturally. The variation of the radius can not be greater than the velocity of the agents otherwise the tracking can not be achieved. Hence a new strategy which tackles the objectives needs to be developed. The velocity $v_{k}$ becomes a new and necessary control input to overcome this mechanical constraint. Then, in the sequel, the variables $\left(v_{k}, u_{k}\right)$ and $\left(\mathbf{r}_{k}, \theta_{k}\right)$, respectively, are the inputs and the state of the agent $k$. In the latter, the notations $\mathbf{r}$ and $\theta$ denote the vectors containing the position and headings of all the agents.

\section{Contraction Control Design}

Consider the problem of tracking a circular formation defined by a constant and known center $\mathbf{c}$ and a timevarying radius. Assume that the radius reference $R_{d}$ is always positive and its first and second time-derivative are known and bounded. Moreover, the following assumption is required:

Assumption 1. Let $t_{s}>0$ be a sufficiently large time to be defined latter. Assume the radius $R_{d}$ satisfies the conditions:

$$
\forall t<t_{s}, \quad R_{d}(t)=R_{0}, \quad \dot{R}_{d}(t)=\ddot{R}_{d}(t)=0
$$

Remark 1 This assumption corresponds to a kind of initialisation of the multi-agent systems. The idea is to permit the agents to reach a circular formation with a constant radius and then to start tracking the time-varying radius reference, as shown in Figure 3. This assumption is not restrictive since this initialization protocol would be used in practice. 
The main contribution of the present article is the introduction of new variable $\hat{\mathbf{r}}_{k}$ such that:

$$
\hat{\mathbf{r}}_{k}=\frac{\tilde{\mathbf{r}}_{k}}{R_{d}}
$$

scaling the relative velocity. This new system of coordinates $\hat{\mathbf{r}}_{k}$ are designed so that it does not depend on the radius reference. The circular motion is normalized also with unit radius then $\left|\dot{\hat{\mathbf{r}}}_{k}\right|=\left|\omega_{0}\right|$. Consider now that the dynamics are driven by:

$$
\dot{\hat{\mathbf{r}}}_{k}=\left|\omega_{0}\right| e^{i \psi_{k}}
$$

where $\dot{\psi}_{k}$ is the only control input to stabilize the new system to the circular motion. Therefore this problem is equivalent to the one treated in Theorem 1.

A new control law is proposed in the following theorem:

Theorem 2 Consider three positive scalars $\epsilon>0$ and $0<R_{1}<R_{2}$. Let $\left|\omega_{0}\right| \neq 0$ and $\kappa>0$ be two control parameters. Let $R_{d}: \mathbb{R} \rightarrow\left[R_{1}, R_{2}\right]$ be a twice differentiable function, with bounded first and second time-derivatives, which satisfies Assumption 1 and the condition:

$$
\forall t, \quad \dot{R}_{d}(t)<\frac{R_{d}(t)\left|\omega_{0}\right|}{(1+\epsilon)}
$$

Then the control law:

$$
\begin{aligned}
v_{k} & =\left|R_{d}\right| \omega_{0}\left|e^{i \psi_{k}}+\frac{\dot{R}_{d}}{R_{d}} \tilde{\boldsymbol{r}}_{k}\right| \\
u_{k} & =\left(1-\frac{\dot{R}_{d}}{R_{d}} \frac{\left\langle\dot{\boldsymbol{r}}_{k}, \tilde{\boldsymbol{r}}_{k}\right\rangle}{v_{k}^{2}}\right) \dot{\psi}_{k}+\frac{\left\langle\tilde{\boldsymbol{r}}_{k}, \dot{\boldsymbol{r}}_{k}\right\rangle}{v_{k}^{2}} \frac{\left(R_{d} \ddot{R}_{d}-2 \dot{R}_{k}^{2}\right)}{R_{k}^{2}} \\
\dot{\psi}_{k} & =\omega_{0}\left(1+\kappa\left\langle\hat{\boldsymbol{r}}_{k}, \dot{\hat{\boldsymbol{r}}}_{k}\right\rangle\right)
\end{aligned}
$$

with the initial conditions $\psi_{k}(0)$ as:

$$
\psi_{k}(0)=\theta_{k}(0)
$$

makes all the agents defined by (1) converge to a circular motion of center $\boldsymbol{c}$, and of radius the time-varying reference $R_{d}$. The direction of rotation is determined by the sign of $\omega_{0}$.

Proof: The convergence to the formation is analyzed with the Lyapunov function:

$$
S(\hat{\mathbf{r}}, \psi)=\frac{1}{2} \sum_{k=1}^{N}\left|\dot{\mathbf{r}}_{k}-i \omega_{0} \hat{\mathbf{r}}_{k}\right|^{2}
$$

Evaluating the derivative of $S(\hat{\mathbf{r}}, \psi)$ along the solutions of the resulting closed-loop system (4) with (6c) leads to:

$$
\begin{aligned}
\dot{S}(\hat{\mathbf{r}}, \psi) & =\sum_{k=1}^{N}\left\langle\dot{\hat{\mathbf{r}}}_{k}-i \omega_{0} \hat{\mathbf{r}}_{k}, i \dot{\hat{\mathbf{r}}}_{k} \dot{\psi}_{k}-i \omega_{0} \dot{\hat{\mathbf{r}}}_{k}\right\rangle \\
& =\left\langle\omega_{0} \hat{\mathbf{r}}_{k},\left|\omega_{0}\right| e^{i \psi_{k}}\right\rangle\left(\omega_{0}-\dot{\psi}_{k}\right) \\
& =-\kappa \sum_{k=1}^{N}\left\langle\omega_{0} \hat{\mathbf{r}}_{k},\left|\omega_{0}\right| e^{i \psi_{k}}\right\rangle^{2} \leq 0
\end{aligned}
$$

Therefore $S(\hat{\mathbf{r}}, \psi)$ is an suitable Lyapunov function for this system. Note that when $S(\hat{\mathbf{r}}, \psi)=0$ the dynamics of agents satisfy $\dot{\hat{\mathbf{r}}}_{k}=i \omega_{0} \hat{\mathbf{r}}_{k}$ which is the kinematic relation for the rotation of the rigid body. Thus, the agents asymptotically reach the circular formation centered at $\mathbf{c}$ and of time-varying radius $R_{d}$ with fixed angular velocity $\left|\omega_{0}\right| \neq 0$.

The next step of the proof concerns the design of the control inputs of the original system. Considering (1a), it is easy to see that $v_{k}$ and $\theta_{k}$ are given by:

$$
v_{k}=\left|\dot{\mathbf{r}}_{k}\right| \quad \text { and } \quad \tan \theta_{k}=\frac{\left\langle\dot{\mathbf{r}}_{k}, i\right\rangle}{\left\langle\dot{\mathbf{r}}_{k}, 1\right\rangle}
$$

Using (3), the control input $v_{k}$ is thus straightforwardly given by (6a). A more particular attention is addressed to the input $u_{k}$. An expression of $\dot{\theta}_{k}$ is obtained by computing the derivative of $\tan \left(\theta_{k}\right)$ as follows:

$$
\dot{\theta}_{k}\left(1+\tan ^{2} \theta_{k}\right)=\frac{d}{d t}\left(\frac{\left\langle\dot{\mathbf{r}}_{k}, i\right\rangle}{\left\langle\dot{\mathbf{r}}_{k}, 1\right\rangle}\right)
$$

or equivalently

$$
\dot{\theta}_{k}=\frac{\left\langle\dot{\mathbf{r}}_{k}, 1\right\rangle^{2}}{\left\langle\dot{\mathbf{r}}_{k}, 1\right\rangle^{2}+\left\langle\dot{\mathbf{r}}_{k}, i\right\rangle^{2}} \cdot \frac{\left\langle\ddot{\mathbf{r}}_{k}, i\right\rangle\left\langle\dot{\mathbf{r}}_{k}, 1\right\rangle-\left\langle\dot{\mathbf{r}}_{k}, i\right\rangle\left\langle\ddot{\mathbf{r}}_{k}, 1\right\rangle}{\left\langle\dot{\mathbf{r}}_{k}, 1\right\rangle^{2}}
$$

Using the properties of the operator $\langle\cdot, \cdot\rangle$, described in the notation, the relation is obtained:

$$
\dot{\theta}_{k}=\frac{\left\langle\ddot{\mathbf{r}}_{k}, i \dot{\mathbf{r}}_{k}\right\rangle}{\left\langle\dot{\mathbf{r}}_{k}, \dot{\mathbf{r}}_{k}\right\rangle}=\frac{\left\langle\ddot{\mathbf{r}}_{k}, i \dot{\mathbf{r}}_{k}\right\rangle}{v_{k}^{2}}
$$

To express this equation in terms of the previous control variable $\dot{\psi}_{k}$ (change of coordinates), note that:

$$
\begin{aligned}
\dot{\mathbf{r}}_{k} & =\dot{\tilde{\mathbf{r}}}_{k}=R_{d} \dot{\hat{\mathbf{r}}}_{k}+\dot{R}_{d} \hat{\mathbf{r}}_{k} \\
\ddot{\mathbf{r}}_{k} & =\ddot{\mathbf{r}}_{k}=i R_{d} \dot{\hat{\mathbf{r}}}_{k} \dot{\psi}_{k}+2 \dot{R}_{d} \dot{\hat{\mathbf{r}}}_{k}+\ddot{R}_{d} \tilde{\mathbf{r}}_{k}
\end{aligned}
$$

Therefore the control law (6b) is obtained.

A control law for the agents to follow a time-varying radius of a circular formation is designed. Note that this control law has singular points when $v_{k}=\left|\dot{\mathbf{r}}_{k}\right|$ is zero. This singular point occurs if there exists a time $t_{c}$ such that:

$$
\begin{aligned}
\angle \tilde{\mathbf{r}}_{k}\left(t_{c}\right) & =-\psi_{k}\left(t_{c}\right), \\
\frac{\left|\dot{R}_{d}\left(t_{c}\right)\right|}{R_{d}\left(t_{c}\right)}\left|\tilde{\mathbf{r}}_{k}\left(t_{c}\right)\right| & =R_{d}\left(t_{c}\right)\left|\omega_{0}\right|
\end{aligned}
$$

To avoid the singular situation when $v_{k}=0$, the initialization protocol described in Assumption 1 is required. Thanks to Theorem 2, the multi-agents system converges asymptotically to the circular formation centered at $\mathbf{c}$ and of radius $R_{0}$. This means that there exists a time $t_{s}$ such that:

$$
\forall t>t_{s}, \quad\left|\hat{\mathbf{r}}_{k}(t)-1\right|=\left|\frac{\tilde{\mathbf{r}}_{k}(t)}{R_{d}(t)}-1\right|<\epsilon
$$

This inequality can be rewritten as $\frac{\left|\dot{R}_{d}\left(t_{c}\right)\right|}{R_{d}\left(t_{c}\right)}\left|\tilde{\mathbf{r}}_{k}\left(t_{c}\right)\right|<$ $\left|\dot{R}_{d}\left(t_{c}\right)\right|(1+\epsilon)$. By vertu of (5), this implies

$$
\forall t>t_{s}, \quad \frac{\left|\dot{R}_{d}(t)\right|}{R_{d}(t)}\left|\tilde{\mathbf{r}}_{k}(t)\right|<R_{d}(t)\left|\omega_{0}\right|
$$

which is in contradiction with (8). Thus the singular point $v_{k}=0$ is avoided. 
Remark 2 Consider the vehicle model (1) with a constant radius of the circle formation $R_{d}=R_{0}$. Then, the the angles $\psi_{k}$ and $\theta_{k}$ are equal, and the control law (6) is the same control as in Theorem 1 with a different $\kappa$ :

$$
v_{k}=R_{0}\left|\omega_{0}\right| \quad \text { and } \quad u_{k}=\dot{\psi}_{k}=\omega_{0}\left(1+\frac{\kappa}{R_{0}^{2}}\left\langle\tilde{\boldsymbol{r}}_{k}, \dot{\boldsymbol{r}}_{k}\right\rangle\right)
$$

Remark 3 In our approach, the angular velocity of the agents $\omega_{0}$ is considered constant, therefore the linear velocity of the agents $v_{k}$ must be time-varying. However, an other control law can be developed for a constant linear velocity of the agents modifying the angular velocity.

\section{Cooperative Control Design Under COMMUNICATION CONSTRAINS}

In the previous section, a control law ensures that the agents reach a circular formation with the time-varying radius $R_{d}$. This section is dedicated to the problem of homogenizing the distribution of the agents along the circle. The case of all-to-all communication and fixed communication graph have already been studied in [11]. Applying this method to the present contraction control law is straightforward, as shown in the similar translation control law developed in [18]. Moreover in practice, considering fixed communication graphs is not realistic since two linked agents could be very far away from one another.

In the case of underwater communication, the quality of the link is strongly affected by the distance between two agents [21]. It might be more interesting to consider distancedependent communication graph. This means that each agent can only receive information from its close neighbors. Hence, a communication area is introduced. Assume this area for any agent is defined by $\rho$ which is the critical communication distance given by the characteristics of the communication devices and of the environment of the AUVs. It is assumed to be the same for all AUVs. The condition to get a communication between $k$ and $j$ is expressed as:

$$
k \in \mathcal{N}_{j} \text { and } j \in \mathcal{N}_{k} \Longleftrightarrow\left|\mathbf{r}_{k}-\mathbf{r}_{j}\right|^{2} \leq(2 \rho)^{2}
$$

Based on the graph theory, a time-varying Laplacian matrix $\mathbf{L}(t)$ is defined as:

$$
L_{k, j}= \begin{cases}d_{k}, & \text { if } k=j \\ -1, & \text { if }\left|\mathbf{r}_{k}-\mathbf{r}_{j}\right| \leq 2 \rho \\ 0 & \text { otherwise }\end{cases}
$$

where $d_{k}$ is the degree of vertex $k$, defined as the number of its neighboring vertices. In such a situation, the following theorem holds.

Theorem 3 Consider two positive scalars $0<R_{1}<R_{2}$ and a twice differentiable function $R_{d}: \mathbb{R} \rightarrow\left[R_{1}, R_{2}\right]$, with bounded first and second time-derivatives. This function satisfies Assumption 1. Let the control parameters be such that $\left|\omega_{0}\right| \neq 0, \kappa>0$, and assuming the condition (5) is satisfied. Let $G(t)$ be the communication graph, $\boldsymbol{L}(t)$

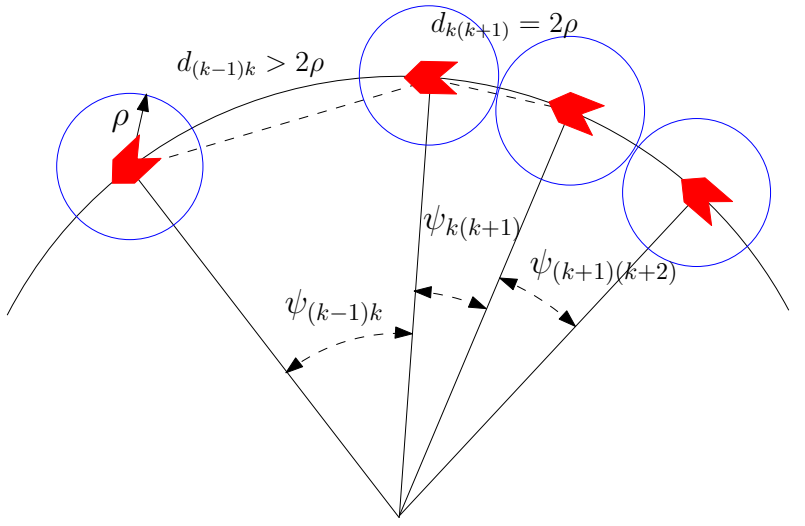

Fig. 2. Formation of communication chains during the contraction motion.

be the corresponding Laplacian matrix and the critical communication distance $\rho$ satisfies:

$$
\rho>R_{2} \sin \frac{\pi}{N}
$$

Then the control law (6) with

$$
\left\{\begin{array}{l}
\dot{\psi}_{k}=\omega_{0}\left(1+\kappa\left\langle\hat{\boldsymbol{r}}_{k}, \dot{\hat{\boldsymbol{r}}}_{k}\right\rangle\right)-\frac{\partial U}{\partial \psi_{k}} \\
U(\psi)=-\frac{K}{N} \sum_{m=1}^{[N / 2]} \frac{1}{2 m^{2}}\left\langle e^{i m \psi}, \boldsymbol{L} e^{i m \psi}\right\rangle
\end{array}\right.
$$

and initial conditions $\psi_{k}(0)$ as:

$$
\psi_{k}(0)=\theta_{k}(0)
$$

ensures that all agents reach the circular formation centered at $\boldsymbol{c}$ of radius $R_{d}(t)$. Moreover the uniform distribution of the agents along the circle is achieved.

Proof: The stability is analyzed by the composed Lyapunov function $V(\hat{\mathbf{r}}, \psi)=\kappa S(\hat{\mathbf{r}}, \psi)+U(\psi)$ whose derivative satisfies $\dot{V}(\hat{\mathbf{r}}, \psi) \leq 0$. Thanks to Theorem 2 , the control law (6) makes all the agents reach the circle centered at $\mathbf{c}$ and radius $R_{d}$. Then, consider the potential function [11]:

$$
U(\psi)=-\frac{K}{N} \sum_{m=1}^{[N / 2]} \frac{1}{2 m^{2}} \sum_{k=1}^{N} U_{m}^{k}(\psi)
$$

where $U_{m}^{k}(\psi)$ can be expresed as:

$$
U_{m}^{k}(\psi)=d_{k}-\sum_{j=1, j \neq k}^{N} L_{k, j}(t) \cos m \psi_{k j}
$$

where $\psi_{k j}=\psi_{k}-\psi_{j}$ denotes the relative angle between agents $k$ and $j$. Note that $\sum_{k=1}^{N} U_{m}^{k}=<e^{i m \psi}, \mathbf{L}(t) e^{i m \psi}>$. The objective of the collaborative control is to minimize the function $U(\psi)$, or equivalently to maximize the functions $U_{m}^{k}(\psi)$ to achieve the uniform distribution. Without loss of generality, consider agent $k . U_{m}^{k}(\psi)$ represents the potential functions associated to agent $k$. The maximum of these functions is obtained when the relative angles between agent $k$ and its neighbors is $\pi / m$. This means that the angle between agent $k$ and its neighbors will tend to $\pi / m$. This works for all $m$ and this finally leads to an increase of the angles between connected agents until the communication 


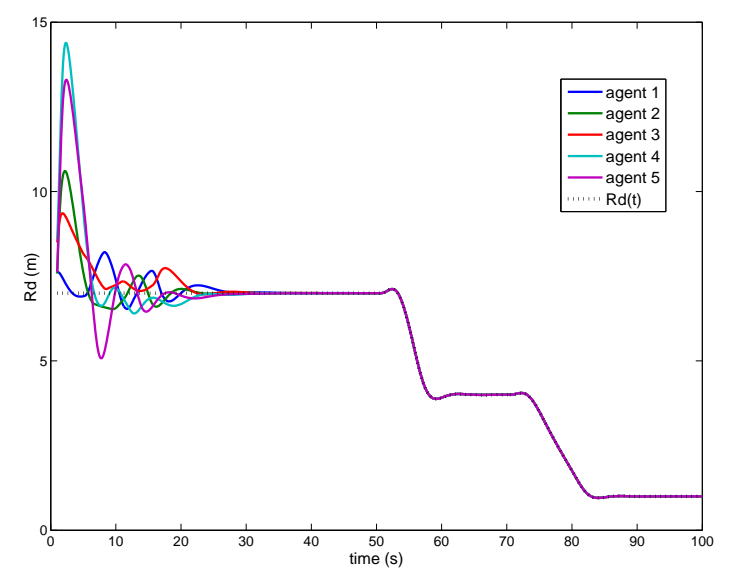

Fig. 3. Simulation of five agents with the controller designed in Theorem 2. The time-varying radius reference (dashed black line) represents the circle contraction from $R_{1}=7 \mathrm{~m}$ to $R_{3}=1 \mathrm{~m}$ crossing $R_{2}=4 \mathrm{~m}$. The color lines represent the distance to center of each agent.

between them is lost. As shown in Figure 2, the geometry of the problem ensures that the connection between agent $k$ and a neighbor $j$ is lost when:

$$
\sin \frac{\psi_{k j}}{2}=\frac{\rho}{R_{d}(t)}
$$

On the other side, $U_{m}^{k}(\psi)$ is discontinuous because of the definition of the Laplacian matrix $\mathbf{L}$. Note that the communication with any agent, for instance $j$, leads to a contribution in the potential function of the following type:

$$
1-\cos \psi_{k j} \geq 0
$$

Thus, if a communication is lost, a positive contribution is removed. Therefore, the potential functions decrease discontinuously. Finally the agents are deployed along the circle. The condition (10) ensures that this expansion guarantees that the agents are connected at least in $d_{0}$-circular graph.

Applying Theorem 7 from [11], the fact that $\mathrm{G}$ is a circular graph implies that the splay pattern, $(N, N)$-pattern, corresponding to the uniform distribution is locally asymptotically stable. No other local critical point is achieved because other critical points of the potential function require that a link between agents is broken and consequently an increase of the potential function. Therefore all the agents are uniformly distributed along the circle. Thanks to change of coordinates (3), the dynamic closed-loop equation corresponding to our approach (time-varying radius) is time-invariant, hence LaSalle principle can be applied.

Remark 4 The set of curve-phase arrangements that are balanced modulo $2 \pi / N$ (uniform distribution) is asymptotically stable for $K>0$. Moreover if $K<0$ the control law of Theorem 3 forces convergence to the synchronized circular formation [11].

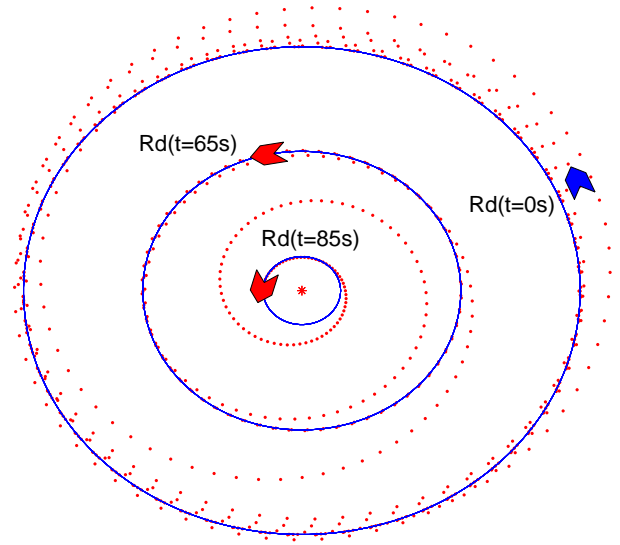

Fig. 4. Simulation of an agent with the control law (6) from Theorem 2. The control law parameters are $\omega_{0}=1$ and $\kappa=1$. The trajectory of the agent (the dashed red line) shows the time-varying radius reference (figure 3 ) followed. The figure shows three snapshots. The blue agent represents the initial condition $R_{d}(0)=7 \mathrm{~m}$. The reds ones represent an intermediate state $R_{d}(65 s)=4 m$ and the final state $R_{d}(85 s)=1 m$.

Remark 5 As in Remark 1, if the reference radius is chosen constant, $R_{d}(t)=R_{0}$, then the control law (10) still holds.

Remark 6 The same framework can also be applied to the case of circular formation control to track a time-varying center [18].

\section{Simulation Results}

This section presents the simulations of the multi-agents system composed of AUVs whose dynamics are defined in (1). In all simulations the controller parameters are $\kappa=1$ and $\omega_{0}=1$. The control parameter to achieve the uniform distribution is $K=0.1$.

Figure 3 shows the evolution in time of the relative position magnitudes $\left|\tilde{\mathbf{r}}_{k}\right|$ of five agents controlled by (6) from Theorem 2. The five agents follow the time-varying radius reference $R_{d}(t)$. This function satisfies Assumption 1 and condition (5).

Figure 4 shows the trajectory of only one agent governed by the control law defined in Theorem 2 during the contraction motion defined by the time-varying radius reference $R_{d}(t)$ from Figure 3 . The tracking of the circle contraction is achieved for any random initial conditions (position and heading of the agent).

Figure 5 shows the evolution of five agents and its distribution along a fixed circle whose radius is constant $R_{0}=5 \mathrm{~m}$. Each figure shows a snapshot from the same simulation. The five agents are governed by the control law defined in Theorem 3. The critical communication distance $\rho=3.5$ satisfies the condition (10). Thanks to the timevarying communication graph is complete, from a certain instant, the agents achieve the uniform distribution all over the circle at $t=30 \mathrm{~s}$. 


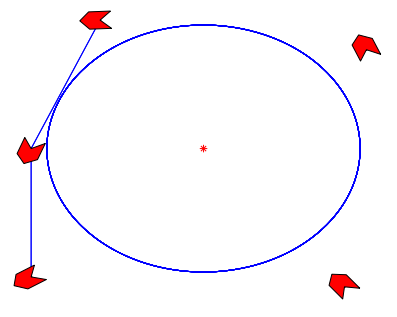

(a) Initial conditions $t=0 \mathrm{~s}$

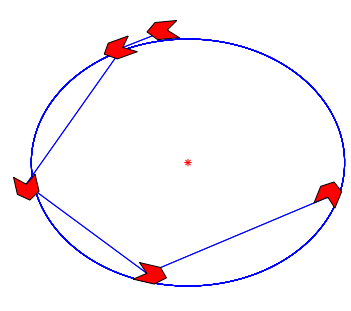

(b) Intermediate state $t=15 \mathrm{~s}$

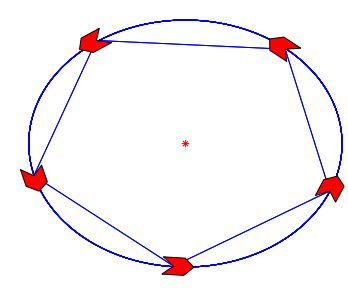

(c) Final uniform distribution state $t=30 \mathrm{~s}$

Fig. 5. Simulation of five agents with the controller designed in Theorem 3 and range-dependent communication. The critical communication radius is $\rho=3.5$. Each figure shows the communication graph represented by the blue lines.

\section{CONCLUSIONS}

We presented a contraction control law that stabilizes a circular formation centered at fixed center and tracking a time-varying radius of the circle. This radius is a given reference which is known for all the agents. Moreover, this paper proposes a cooperative control algorithm to achieve the uniform distribution of the agents along the circular formation. This algorithm integrates with the contraction control a potential function which reaches its minimum in the desired uniform configuration. This potential function is designed taking into account the communication constraints between agents. The result of this combination is a cooperative control of a planar particle model with limited communication to track a time-varying reference. The obvious following step is combined the translation [18] and contraction laws in order to control the main transformations of the circle.

At this time, it is assumed that all agents have perfect knowledge of the position of the fixed center $\mathbf{c}$ and of the radius reference $R_{d}$ and its first and second derivatives. One can consider this assumption as a very restrictive one. However, this constitues a first step of our research and further developments would consider a cooperative algorithm which will avoid this assumption.

\section{ACKNOWLEDGEMENTS}

This research is supported by FeedNetBack project and CONNECT project. FeedNetBack is a recently accepted EU STREP project FP7-ICT-2007-2 and the CONNECT project is funded by ANR (Agence Nationale de la Recherche), PSIROB06-174215

\section{REFERENCES}

[1] R. Olfati-Saber, J. A. Fax, and R. M. Murray, "Consensus and cooperation in networked multi-agent systems," Proceedings of the IEEE, vol. 95, no. 1, pp. 215-233, January 2007.

[2] N. E. Leonard, D. A. Paley, F. Lekien, R. Sepulchre, D. M. Frantatoni, and R. E. Davis, "Collective motion, sensor networks and ocean sampling," Proceedings of the IEEE, vol. 95, no. 1, pp. 48-74, January 2007.

[3] E. Fiorelli, P. Bhatta, N. E. Leonard, and I. Shulman, "Adaptive sampling using feedback control of an autonomous underwater glider fleet," Proceedings of 13th Int. Symp. on Unmanned Untethered Submersible Technology (UUST), August 2003.
[4] R. L. Raffard, C. J. Tomlin, and S. P. Boyd, "Distributed optimization for cooperative agents: Application to formation flight," $43 \mathrm{rd}$ IEEE Conference on Decision and Control, 2004.

[5] R. Olfati-Saber and R. M. Murray, "Consensus problems in networks of agents whit switching topology and time-delays," IEEE Transactions on Automatic Control, vol. 49, no. 9, pp. 1520-1533, September 2004.

[6] D. A. Paley, N. E. Leonard, and R. Sepulchre, "Stabilization of symmetric formations to motion around convex loops," Systems \& Control Letters, vol. 57, pp. 209-215, 2008.

[7] R. Sepulchre, D. A. Paley, and N. E. Leonard, "Stabilization of planar collective motion: All-to-all communication," IEEE Transactions on Automatic Control, vol. 52, pp. 811-824, 2007.

[8] R. Olfati-Saber, "Flocking for multi-agent dynamic systems: Algorithms and theory," IEEE Transactions on Automatic Control, vol. 51, no. 3, p. 401420, March 2006.

[9] P. Ögren, E. Fiorelli, and N. E. Leonard, "Cooperative control of mobile sensor networks: Adaptive gradient climbing in a distributed environment," IEEE Transactions on Automatic Control, vol. 49, August 2004.

[10] D. V. Dimarogonas and K. J. Kyriakopoulos, "On the rendezvous problem for multiple nonholonomic agents," IEEE Transactions on Automatic Control, vol. 52, May 2007.

[11] R. Sepulchre, D. A. Paley, and N. E. Leonard, "Stabilization of planar collective motion with limited communication," IEEE Transactions on Automatic Control, vol. 53, pp. 706-719, 2008.

[12] S. Martínez, J. Cortés, and F. Bullo, "Motion coordination with distributed information," IEEE Control Systems Magazine, 2007.

[13] P. Ögren, M. Egerstedt, and X. Hu, "A control lyapunov function approach to multiagent coordination," IEEE Transactions on Automatic Control, vol. 18, no. 5, October 2002.

[14] R. Sepulchre, D. Paley, and N. E. Leonard, "Graph laplacian and lyapunov design of collective planar motions," Proceedings of International Symposium on Nonlinear Theory and its Applications, 2005.

[15] C. W. Reynolds, "Flocks, herds, and schools: A distributed behavioral model," in Computer Graphics, vol. 21, no. 4, July 1987.

[16] N. E. Leonard and E. Fiorelli, "Virtual leaders, artifitial potentials and coordinated control of goups," Proceedings of 40st IEEE Conf. Decision and Control, 2001.

[17] W. Ren and R. W. Beard, "Consensus seeking in multi-agents systems under dynamically changing interaction topologies," IEEE Transactions on Automatic Control, vol. 50, pp. 655-661, 2005.

[18] L. Briñón, A. Seuret, and C. Canudas, "Translation control of a fleet circular formation of auvs under finite communication range," accepted for the 48th IEEE Conference on Decision and Control, 2009.

[19] Z. Lin, B. Francis, and M. Maggiore, "Necessary and sufficient graphical conditions for formation control of unicycles," in IEEE Transactions on Automatic Control, 2007.

[20] R. Sepulchre, D. A. Paley, and N. E. Leonard, "Group coordination and cooperative control of steered particles in the plane," Lecture Notes in control and Information Sciences, 2006.

[21] M. Stojanovic, "Recent advances in high rate underwater acoustic communications," IEEE J. Oceanic Eng., 1996. 